\title{
Schedule Risk Analysis using a Proposed Modified Variance and Mean of the Orig- inal Program Evaluation and Review Technique Model
}

\author{
Solomon Sackey* and Byung-Soo Kim**
}

Received September 12, 2018/Accepted November 22, 2018/Published Online January 14, 2019

\begin{abstract}
The Program Evaluation and Review Technique (PERT) model uses parameters such as the specified project completion time, mean, and variance to estimate the probability of project completion time. However, this model uses a weighted average and unweighted value in the variance, which is based on six sigma of the mean. Despite many proposed modifications to improve the traditional PERT model, the hidden error in the calculation of the variance and mean of the PERT approach has not been adequately addressed. This error leads to underestimation of the schedule risk. Considering the impact of variance and mean on the probability of project completion times, this study contributes to the improvement of the accuracy of schedule risk estimation by proposing a modified variance and mean of the original PERT model. The original PERT model was first used to estimate the project completion time. However, using the proposed modified model to estimate the completion time, a $95 \%$ confidence interval assumption and the corresponding distribution within \pm 2 standard deviation of the mean and standard or $Z$ values were employed to model the new mean and variance equations. To prove the validity of the proposed modified variance and mean assumptions, we performed a schedule risk analysis through simulation using Oracle Crystal Ball for comparison. The results showed that the proposed PERT model had a better mean error rate of $2.46 \%$ as compared to $3.31 \%$ of the original PERT model.
\end{abstract}

Keywords: simulation, PERT, schedule risk analysis, project completion times, probability

\section{Introduction}

Program Evaluation and Review Technique (PERT) is one of the two contemporary project planning and scheduling approaches used by project management teams in different fields, not limited to construction. PERT was first proposed in the late 1950s by (Malcolm et al., 1959) as a project management technique that relied mainly on beta distribution owing to its simplicity and flexibility. The PERT was developed for the U.S. Navy's Polaris project having thousands of contractors with the potential to reduce both the time and cost required to complete the project. The introduction of this method gained popularity for its application in engineering and research development program of the Polaris missiles. Since its introduction over 50 years ago, it has been applied to different fields, including but not limited to construction projects, research development, installation of new computer systems, and product development. PERT plays an important role in project management and represents a quantum shift in the practice of project planning and scheduling.

Unlike the Critical Path Method (CPM) model, which uses deterministic durations, the PERT approach uses a three-point time estimate, which considers the uncertainties of the activity durations. After identifying all the activities in the project and providing the respective relationships among them, the duration of these activities is modeled (Ballesteros-Pérez, 2017). Project planners have been familiar with PERT as a standard scheduling and project control approach for many years (Dawson, 1998). One objective of the PERT model is to assist a project manager to identify bottlenecks and overruns in the project before they happen so that corrective actions can be taken before it is too late to effect any change (Van Slyke, 1963). According to (Jun et al., 2006), the PERT technique "evaluates the lead time of a project under the assumption that each activity has a stochastic duration time." Similar to the CPM model, the PERT technique estimates the project completion time based on the expected duration of the activities on the critical path. Schedule risk analysis is performed to assess the criticality of the activities and also to estimate the probability of project completion times.

This study focused on tackling an important area of the original PERT technique. The study investigated and identified the hidden error in the calculation of the variance and mean of the PERT model and proposed a modified variance and mean model to improve the accuracy of the estimate of project completion times. The variance is very useful in estimating the probability of project completion times based on the PERT model. It measures the deviation of the time of individual activities from the mean. Further, the variance can be defined in the scope of project management as the sum of the squares of the deviation in the

\footnotetext{
*Ph.D. Candidate, Dept. of Civil Engineering, Kyungpook National University, Daegu 41566, Korea (E-mail: s.sackey123@knu.ac.kr)

**Member, Professor, Dept. of Civil Engineering, Kyungpook National University, Daegu 41566, Korea (Corresponding Author, E-mail: bskim65@knu.ac.kr)
} 
activity durations. To further emphasize the importance of variance in project completion on time, one of the assumptions of the PERT model is that, if there are two or more critical paths in the activity network, the part with the largest variance is chosen as the critical path. However, the hidden error in the variance formula is that the PERT model uses an unweighted value in the variance, which is based on 6-sigma of the mean. This leads to underestimation of the variance of the activities, which affects the accuracy of the probabilities of completing the project within a specified time. It may seem practical but is unrealistic to estimate the range values of the optimistic and pessimistic time durations based on the $99.7 \%$ confidence level. This indicates that less than $0.5 \%$ falls outside the lower and upper limits. The range of values is merely an estimate, and hence, there must be uncertainties related to it. It cannot be an accurate estimate. The newly proposed variance and mean are calculated based on a 95\% confidence level assumption, which a project management team may find comfortable for providing estimates.

The PERT literature is applied to help understand the theoretical aspect of the original PERT approach and to also understand the importance of its application to project management. Since its introduction, the PERT approach has been studied extensively. PERT assumes that activity duration is a random variable that can be derived through the use of a simple formula (Lee et al., 2013). A previous study (Miklos Hajdu and Bokor, 2014) investigated the effects of different activity distributions on the project duration in PERT networks and stated that activity durations are defined by stochastic variables that are to be independent of each other in the PERT network. In the PERT method, the Research and Development (R and D) program are characterized as a network of interrelated events to be accomplished in a properly ordered sequence (Malcolm et al., 1959). Another report (Trietsch and Baker, 2012) presented a study on fitting PERT/CPM for use in the $21^{\text {st }}$ century. Providing a contrast to the CPM, the authors stated that the PERT focuses on creating and controlling project schedules in a stochastic environment. A similar study (Herrerías-Velasco et al., 2011) examined the revisiting of the PERT mean and variance. A previous study (Hajdu and Bokor, 2016) investigated whether activity duration distribution is important in a sensitivity analysis in PERT networks. The PERT method has many advantages, one of which is mentioned in a previous report (Mishakova et al., 2016) i.e., that the PERT method allows calculating the probability of completing the project on time. Another report (Udoumoh and Ebong, 2017) provides the advantage of using the PERT method. The authors stated that the PERT method is not only useful in determining the project completion times but also workable and cost-effective to manage projects.

Despite its usefulness in project management, the original PERT has received several criticisms from researchers and practitioners. Several improvement models and formula modifications have been proposed since its introduction. The study by Trietsch et al. (2012) criticized that the PERT-beta distribution only relies on subjective estimates. The authors proposed a new scheduling framework called "PERT 21" using Parkinson distribution with the lognormal core. The study by (Jun et al., 2006) also argued that the PERT model cannot handle complex patterns among activities. Similarly, the study by (Park and Cutkosky, 1999) argued that the PERT model was designed for linear processes and thus, it cannot represent circuits. Owing to these limitations and to the fact that the PERT model assumes the range of distribution to cover 6 standard deviations, a modification is required.

The study by (Vanhoucke, 2012) defines schedule risk analysis as a "technique to measure the sensitivity of project activities and to predict the expected influence of variability in activity duration/ costs on the project objective." As the probability of completing most projects within a specified time period is always difficult to determine (Lee, 2005), developed a stochastic project-scheduling simulation software to measure the probability of completing a project within a specified time period. In another study (Reis \& Lucko, 2016) investigated a productivity method with maximum constraints by developing an algorithm to improve the scheduling method. There are several risks and uncertainties existing in projects. To overcome these possible uncertainties, (Razaque et al., 2012) used fuzzy failure mode and effect analysis to analyze the potential risk critically. More so, Avlijas (2018) studied the use of Monte Carlo simulation as a method for the analysis of the risks that impact project duration. In a more different approach, (Mccabe, 2003) developed a probabilistic model to translate project characteristics into schedule risk boundaries.

\section{Methodology and Procedure}

The PERT model is one of the most preferred project planning and scheduling approaches used by project managers. However, this model has certain drawbacks and limitations, which require modifications. The authors used the original PERT model to estimate the expected total project completion time and analyzed the probability of completing the project within that estimated time. The PERT model uses the three-point estimate to determine the mean duration of each activity in the project. The mean durations were calculated as follows:

Mean $(\mu)=\left[T_{O}+\left(4 T_{M}\right)+T_{P}\right] / 6$

The mean can also be simplified as follows:

Mean $(\mu)=T_{O} / 6+2 / 3 T_{M}+T_{P} / 6$

$\mu=0.1667 T_{O}+0.667 T_{M}+0.167 T_{P}$

Further, the standard deviation and variance were calculated as follows:

Standard deviation $(\sigma)=\left(T_{P}-T_{O}\right) / 6$

Variance $\sigma^{2}=\left[\left(T_{P}-T_{O}\right) / 6\right]^{2}$

where,

$T_{O}$ is the optimistic time,

$T_{M}$ is the most likely time, and

$T_{P}$ is the pessimistic time.

The proposed modification model is based on an assumption 
of $95 \%$ confidence level. In statistics and probability, the common confidence levels include $90 \%, 95 \%$, and $99 \%$. Even though different confidence level could be investigated, the authors used $95 \%$ confidence level to modify the original PERT model based on the fact that $95 \%$ confidence interval is the conventional confidence level that most decision makers or project managers are comfortable dealing with. Project planners or managers use forecast estimates to plan the project and with a high degree of certainty that the project would be completed before the predicted expected completion time or within the lower and upper time interval. It is reasonable to adopt $95 \%$ confidence interval in that with just $5 \%$ risk that the expected project duration might deviate from the true mean duration, the management team has a high confidence of 0.95 probability that the project would be completed within the predicted mean duration. The authors proposed that the three beta-distribution parameters (minimum, most likely and maximum) durations should be obtained at $95 \%$ confidence level. In other words, there is a $95 \%$ probability that the activity duration will be within the optimistic and pessimistic range with respect to a standard normal distribution. There is a $5 \%$ probability that the activity duration will be less than the lower or exceeds the upper bounds. Hence, the authors proposed the modified PERT model equations as follows:

$$
\text { Mean }(\mu)=0.025 T_{O}+0.95 T_{M}+0.025 T_{P} \text {. }
$$

For the variance, the corresponding interval value for a $95 \%$ confidence level was used. For a normal distribution, the equivalent critical value is $1.96 \sigma$; twice this value yields 3.92 . The factor 1.96 is based on the assumption that $95 \%$ of the area under normal distribution is within $1.96 \sigma$ of the mean. From the $Z$ score table, the corresponding $\mathrm{Z}$ value for $95 \%$ is 1.64 .

Therefore, the variance was proposed as follows:

$$
\begin{aligned}
\text { Variance } \sigma^{2} & =\left[\left(T_{P}-T_{O}\right) /\left(\mathrm{SD}_{0.95} \times \text { standard or } \mathrm{Z} \text { score }\right)\right]^{2} \\
\text { Variance } \sigma^{2} & =\left[\left(T_{P}-T_{O}\right) / 3.92 \times 1.64\right]^{2} \\
\text { Variance } \sigma^{2} & =\left[\left(T_{P}-T_{O}\right) / 6.43\right]^{2}
\end{aligned}
$$

In Eq. (7), $\mathrm{SD}_{0.95}$ refers to the $95 \%$ of the distribution within \pm 2 standard deviation of the mean, which has a theoretical value of 1.96 on each side of the normal distribution curve. The standard or $\mathrm{Z}$ score refers to the standard score corresponding to 0.95 probability using the standard normal distribution table. We, therefore, calculated a single critical value for the $95 \%$ confidence interval by multiplying the 2 standard deviation values on each side of the curve and the approximate standard score on the $Z$ table. $Z$ scores are used to calculate the probability of a score within a normal distribution. However, if the probability is known, the $\mathrm{Z}$ score could alternatively be found. Fig. 1 shows the critical value of $95 \%$ confidence level under the normal distribution. It shows that $95 \%$ of the area under the normal distribution curve is $1.96 \sigma$ of the mean.

Beta-PERT distribution was used to generate a random duration for each activity. The three parameters used in beta-pert distribution are the minimum, most likely (mode) and the maximum duration to model a smooth curve that fits very well to the normal

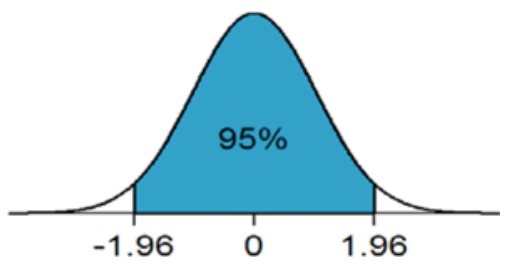

Fig. 1. The Critical Value of $95 \%$ Confidence Level

distribution. The range values, minimum and the maximum duration are used to account for the uncertainties in the activity duration. Crystal Ball was used as the simulation tool to perform Monte Carlo simulation on the expected project duration determined from the start and finish dates of the individual activities. Although schedule risk analysis was conducted, the authors focused only on analyzing the probability of completing a project within a specified time duration and the sensitivity measure analysis of the activities. Regarding the research procedure and process, activities of the project under the study were first defined. Fig. 2 illustrates the process of the study via a flowchart. Each activity was thereafter assigned a three-point time estimate and the mean and variance were calculated. The critical path through the network was determined and the expected project completion times were estimated. The respective project completion probabilities were estimated for the original PERT model and the proposed modified PERT model. Using beta-PERT distribution, the random duration for each activity was generated and a simulation was performed to assess the real probability of completing the project.

\section{Probability Network Analysis}

The network diagram shown in Fig. 3 illustrates the relationship between the activities of a simple project. The individual tasks are represented by alphabetical initials. Using the typical forward and backward pass approach, the critical path was identified as

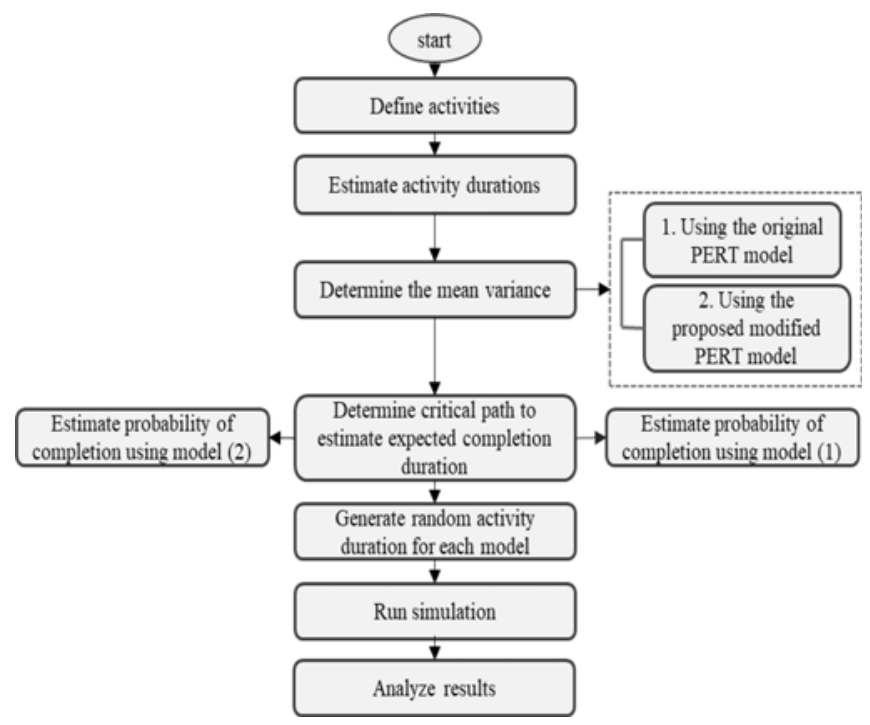

Fig. 2. Research Procedure and Process 
(A--D--F--G). The network was used to analyze both the original PERT model and the proposed modified PERT model. Thus, there are 7 major activities and to estimate the probability of completion, Excel function NORM.DIST (Due date, mean, SQRT (variance), TRUE) was deployed.

\subsection{Estimation of Project Completion Time-the Original PERT Model}

The expected project duration and the probability of completion were estimated using the original PERT model with Eqs. (1) and (5), respectively. The calculation is illustrated in Table 1. It presents the activities, precedence activities, three-point estimate, and estimation of the mean and variance. The probability of completing the estimated project duration was calculated using a specified project completion time and the sum of variance of activities on the critical path. The expected duration and the variance were calculated by adding the mean durations and the corresponding variance of the activities on the critical path. The simulation was performed to determine the real probability of completing the project within the expected duration as depicted in Fig. 4. In performing the simulation in Crystal Ball, first, a probability distribution assumption was defined as beta-PERT distribution for all the activities. Second, based on the activity relationships, the start and finish times were estimated and random activity durations were generated to determine the expected project duration. This expected duration becomes the target forecast. Thus, Crystal Ball uses this target forecast to simulate the project to produce output results.

\subsubsection{Simulation Analysis of the Original PERT Model}

The simulation results of applying the original PERT method using the Crystal Ball application is shown in Fig. 4. It shows the mean or the expected duration of the project through the CPM and the certainty level or the probability of completing within the specified due date.

\subsection{Estimation of Project Completion Time-the Proposed Modified PERT model}

Probability network analysis for the proposed modified PERT model was performed in a similar manner. However, as the authors

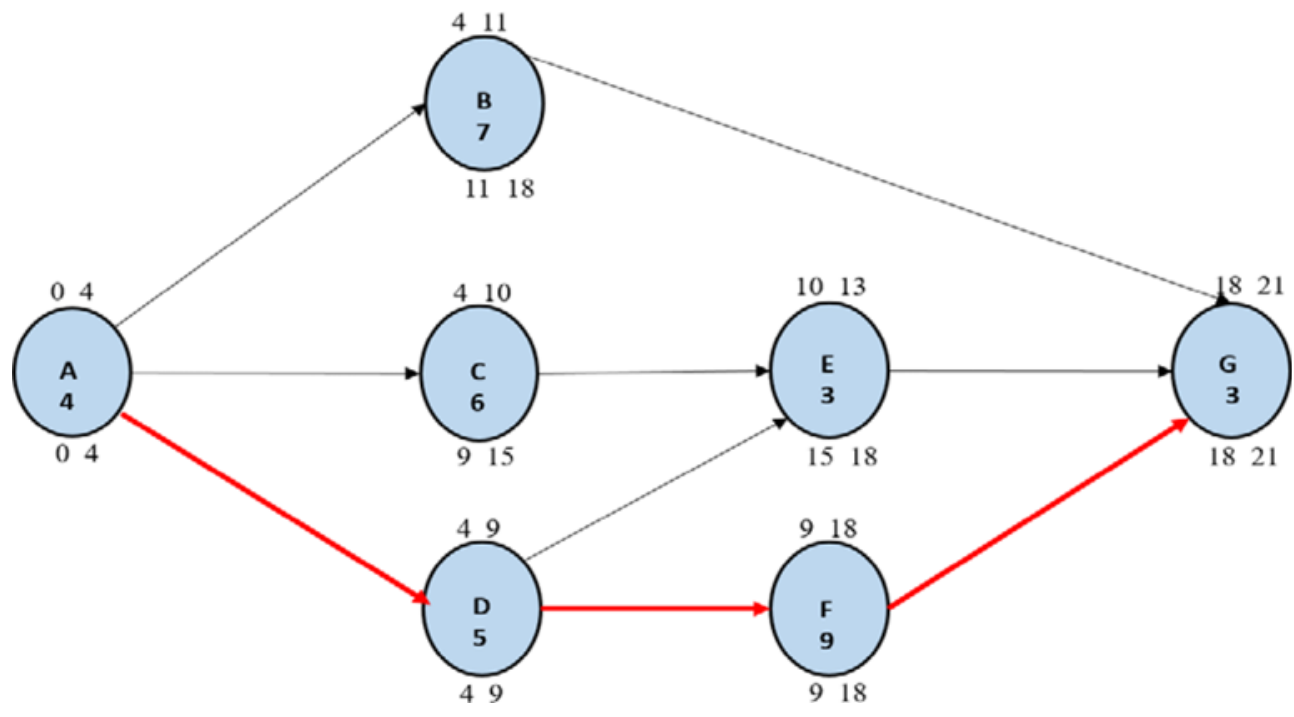

Fig. 3. Project's Network Diagram

Table 1. Probability Network Analysis-the Original PERT Model

\begin{tabular}{|c|c|c|c|c|c|c|}
\hline Activities & Predecessors & Optimistic time & Most Likely time & Pessimistic time & Mean duration & Variance \\
\hline $\bar{A}$ & & 2 & 4 & 7 & 4.17 & 0.69 \\
\hline B & A & 5 & 7 & 11 & 7.33 & 1.00 \\
\hline $\mathrm{C}$ & A & 4 & 6 & 10 & 6.33 & 1.00 \\
\hline $\bar{D}$ & $\overline{\mathrm{A}}$ & 3 & 5 & 9 & 5.33 & 1.00 \\
\hline $\mathrm{E}$ & C, D & 2 & 3 & 5 & 3.17 & 0.25 \\
\hline$F$ & $\mathrm{D}$ & 6 & 9 & 14 & 9.33 & 1.78 \\
\hline G & $\mathrm{B}, \mathrm{E}, \mathrm{F}$ & 2 & 3 & 5 & 3.17 & 0.25 \\
\hline Project end & G & 0 & 0 & 0 & 0.00 & 0.00 \\
\hline & Critical path & Expected duration & Variance & Due date & \multicolumn{2}{|c|}{ Prob. of completion } \\
\hline & A --D--F--G & 22.00 & 3.72 & 23weeks & \multicolumn{2}{|c|}{$69.79 \%$} \\
\hline & & & \multicolumn{2}{|c|}{ Probability of delay } & \multicolumn{2}{|c|}{$30.21 \%$} \\
\hline
\end{tabular}




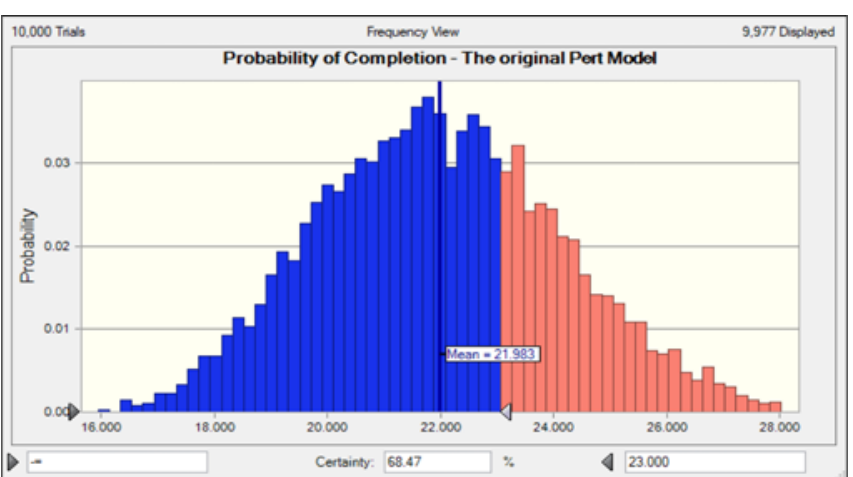

Fig. 4. The Probability of Completion through Simulation-PERT Model

assumed that range values should be estimated with a $95 \%$ confidence level, the optimistic and pessimistic range values were estimated using the assumption of $5 \%$ probability outside which the duration will fall. The calculation basis is illustrated in Table 2 . The estimated optimistic and pessimistic range values for each activity, as used in the original PERT model, were multiplied by $2.5 \%(0.025)$ each. The mean and variance were calculated using Eqs. (6) and (9), respectively.

\subsubsection{Validation of the Proposed Modified PERT Model through Simulation}

To validate the proposed modified PERT model, a similar simulation was performed using the Crystal Ball application to determine the real probability of completing within the specified due date and the results are shown in Fig. 5.

Four other due dates were analyzed as summarized in shown in Table 3: the probability of finishing within 1 week before the expected duration, and 1 week, 2 weeks, and 3 weeks after the expected duration. In each case, the probability of completion and probability of delay were estimated and compared against the simulation results. The error rate for each model was calculated and analyzed. The error rate was calculated as follows:

Error Rate $(\%)=($ SPD - PERT PD $)$

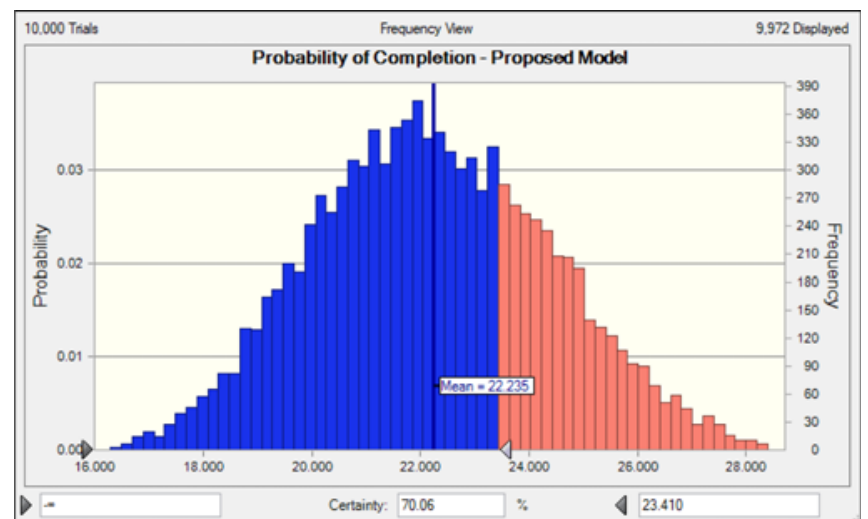

Fig. 5. The Probability of Completion through Simulation-proposed Model

where SPD is the simulation probability of delay, and PERT PD is the probability of delay in the PERT model.

To provide more insight into the validity and effectiveness of the proposed modified PERT model, four other project case studies were analyzed in a similar manner as in case study 1 and the results are summarized in the following tables. In each case study, the original PERT model and the proposed model were analyzed and compared with the simulation results. Case study 2 consists of a project with 10 activities and using the original PERT model, the expected duration was estimated as 24.33 weeks with a variance of 5.44 weeks, whereas the expected duration was 24.54 weeks with a variance of 4.98 weeks using the proposed modified model. Case study 3 is a 4 -unit pupil classroom block project consisting of 11 major activities. Using the original PERT model, the expected duration was estimated to be 25.67 weeks, with a variance of 5.94 weeks, whereas the proposed model estimated the mean to be 26.28 weeks with a variance of 5.35 weeks. Case study 4 is a 2-block residential apartment project consisting of 14 major activities. The estimated project duration and variance using the original PERT were 41.33 weeks and 5.19 weeks, respectively, whereas the proposed model estimated the expected duration as 42.17 weeks with a variance of 4.78 weeks. Case study 5 consists of a project with 9 detailed activities and using

Table 2. Probability Network Analysis-the Proposed Modified PERT Model

\begin{tabular}{|c|c|c|c|c|c|c|}
\hline Activities & Predecessors & Optimistic time & Most likely time & Pessimistic time & Mean duration & Variance \\
\hline A & & 2.05 & 4 & 7.18 & 4.26 & 0.64 \\
\hline B & A & 5.13 & 7 & 11.28 & 7.47 & 0.91 \\
\hline $\mathrm{C}$ & A & 4.10 & 6 & 10.25 & 6.42 & 0.91 \\
\hline $\mathrm{D}$ & $\mathrm{A}$ & 3.08 & 5 & 9.22 & 5.37 & 0.91 \\
\hline $\mathrm{E}$ & C, D & 2.05 & 3 & 5.12 & 3.21 & 0.23 \\
\hline $\mathrm{F}$ & $\mathrm{D}$ & 6.15 & 9 & 14.35 & 9.58 & 1.63 \\
\hline $\mathrm{G}$ & $\mathrm{B}, \mathrm{E}, \mathrm{F}$ & 2.05 & 3 & 5.12 & 3.21 & 0.23 \\
\hline Project end & $\mathrm{G}$ & 0 & 0 & 0 & 0.00 & 0.00 \\
\hline & Critical path & Expected duration & Variance & Due date & \multicolumn{2}{|c|}{ Prob. of completion } \\
\hline & A --D--F--G & 22.41 & 3.40 & 23.41weeks & \multicolumn{2}{|c|}{$70.61 \%$} \\
\hline & & & \multicolumn{2}{|c|}{ Probability of delay } & \multicolumn{2}{|c|}{$29.39 \%$} \\
\hline
\end{tabular}


the original PERT model, the expected duration was estimated as 27.17 weeks with a variance of 4.19 weeks, whereas the proposed modified model estimated the mean as 27.68 weeks with a variance of 3.72 weeks. The proposed model had an average error rate of $1.81 \%$ for all the due dates as compared to $2.48 \%$ of the original PERT with a difference of 0.67 percentage points or $27.02 \%$ [ $(0.68 /$ $2.48)^{*} 100$ ] in case 1 . For case study 2 , the proposed model had a better average error rate of $2.58 \%$ as compared to $3.53 \%$ of the original PERT, with the proposed model performing better by 0.95 percentage points or $26.91 \%$. For case study 3 , the proposed model had an average error rate of $2.50 \%$ as compared to $3.34 \%$ of the original PERT, with the proposed model performing better by 0.84 percentage points or $25.15 \%$. In the case of case study 4 , the original PERT model had an average error rate of $2.47 \%$ as compared to $3.94 \%$ of the proposed model, and hence, the proposed model performs 1.0 percentage points or $25.38 \%$ better. For case study 5 , the proposed model had an average error rate of $2.47 \%$ as compared to $3.24 \%$ of the original PERT, with the proposed model performing better by 0.77 percentage points or $23.77 \%$. Tables 3-7 present the complete output of the analysis. The due dates in the left and right sides of Table 3-7 are different because the mean or expected duration for both the original PERT model and the proposed modified PERT model were calculated from different equations. In the case of the original PERT model, the expected duration was estimated as 22 weeks, and 22.41 weeks in the case of the proposed modified PERT model. However, the same one week chance of completing before, and one, two and three weeks chance of completing after these expected durations were used for the analysis in both models.

In Table 8, the error rates of all the five cases are summarized. It presents the average error rate under each case study where the mean error rate was computed for both the original PERT model and the proposed model.

\subsubsection{Sensitivity Measure Analysis}

Using the forward and backward pass approach in traditional

Table 3. Comparative Analysis of Accuracy Rate/Error Rate-Case Study 1

\begin{tabular}{|c|c|c|c|c|c|c|c|c|c|c|c|}
\hline \multirow{2}{*}{$\begin{array}{l}\text { Due } \\
\text { dates }\end{array}$} & \multicolumn{2}{|c|}{ Simulation (\%) } & \multicolumn{2}{|c|}{ Original PERT model (\%) } & \multirow{2}{*}{$\begin{array}{c}\text { Error rate } \\
\%\end{array}$} & \multirow{2}{*}{$\begin{array}{l}\text { Due } \\
\text { dates }\end{array}$} & \multicolumn{2}{|c|}{ Simulation (\%) } & \multicolumn{2}{|c|}{ Proposed model (\%) } & \multirow{2}{*}{$\begin{array}{c}\text { Error rate } \\
\%\end{array}$} \\
\hline & On time & Delay & On time & Delay & & & On time & Delay & On time & Delay & \\
\hline 21 & 33.64 & 66.36 & 30.21 & 69.79 & 3.43 & 21.41 & 27.89 & 72.11 & 29.39 & 70.61 & 1.50 \\
\hline 23 & 68.47 & 31.53 & 69.79 & 30.21 & 1.32 & 23.41 & 70.06 & 29.94 & 70.61 & 29.39 & 0.55 \\
\hline 24 & 82.13 & 17.87 & 85.00 & 15.00 & 2.87 & 24.41 & 83.08 & 16.92 & 86.09 & 13.91 & 3.01 \\
\hline \multirow[t]{2}{*}{25} & 91.18 & 8.28 & 94.00 & 6.00 & 2.28 & 25.41 & 92.65 & 7.35 & 94.81 & 5.19 & 2.16 \\
\hline & & & Average & & $2.48 \%$ & & & & Average & & $1.81 \%$ \\
\hline
\end{tabular}

Table 4. Comparative Analysis of Accuracy Rate/Error Rate-Case Study 2

\begin{tabular}{|c|c|c|c|c|c|c|c|c|c|c|c|}
\hline \multirow{2}{*}{$\begin{array}{l}\text { Due } \\
\text { dates }\end{array}$} & \multicolumn{2}{|c|}{ Simulation (\%) } & \multicolumn{2}{|c|}{ Original PERT model (\%) } & \multirow{2}{*}{$\begin{array}{c}\text { Error rate } \\
\%\end{array}$} & \multirow{2}{*}{$\begin{array}{l}\text { Due } \\
\text { dates }\end{array}$} & \multicolumn{2}{|c|}{ Simulation (\%) } & \multicolumn{2}{|c|}{ Proposed model (\%) } & \multirow{2}{*}{$\begin{array}{c}\text { Error rate } \\
\%\end{array}$} \\
\hline & On time & Delay & On time & Delay & & & On time & Delay & On time & Delay & \\
\hline 23.33 & 31.89 & 68.11 & 33.36 & 66.64 & 1.47 & 23.54 & 31.96 & 68.04 & 32.70 & 67.30 & 0.74 \\
\hline 25.33 & 62.08 & 37.92 & 66.54 & 33.46 & 4.46 & 25.54 & 65.28 & 34.72 & 67.30 & 32.70 & 2.02 \\
\hline 26.33 & 75.74 & 24.26 & 80.39 & 19.61 & 4.65 & 26.54 & 76.93 & 23.07 & 81.50 & 18.50 & 4.58 \\
\hline \multirow[t]{2}{*}{27.33} & 86.53 & 13.47 & 90.05 & 9.95 & 3.52 & 27.54 & 88.12 & 11.88 & 91.10 & 8.90 & 2.98 \\
\hline & & & Average & & $3.53 \%$ & & & & Average & & $2.58 \%$ \\
\hline
\end{tabular}

Table 5. Comparative Analysis of Accuracy Rate/Error Rate-Case Study 3

\begin{tabular}{|c|c|c|c|c|c|c|c|c|c|c|c|}
\hline \multirow{2}{*}{$\begin{array}{l}\text { Due } \\
\text { dates }\end{array}$} & \multicolumn{2}{|c|}{ Simulation (\%) } & \multicolumn{2}{|c|}{ Original PERT model (\%) } & \multirow{2}{*}{$\begin{array}{c}\text { Error rate } \\
\%\end{array}$} & \multirow{2}{*}{$\begin{array}{l}\text { Due } \\
\text { dates }\end{array}$} & \multicolumn{2}{|c|}{ Simulation (\%) } & \multicolumn{2}{|c|}{ Proposed model (\%) } & \multirow{2}{*}{$\begin{array}{c}\text { Error rate } \\
\%\end{array}$} \\
\hline & On time & Delay & On time & Delay & & & On time & Delay & On time & Delay & \\
\hline 24.67 & 31.91 & 68.09 & 34.13 & 65.87 & 2.28 & 25.28 & 31.70 & 68.30 & 33.30 & 66.70 & 1.60 \\
\hline 26.67 & 62.58 & 37.42 & 65.97 & 34.03 & 3.39 & 27.28 & 64.98 & 35.02 & 66.70 & 33.30 & 1.69 \\
\hline 27.67 & 75.71 & 24.29 & 79.44 & 20.56 & 3.73 & 28.28 & 77.52 & 22.48 & 80.70 & 19.30 & 3.18 \\
\hline \multirow[t]{2}{*}{28.67} & 85.13 & 14.87 & 89.10 & 10.90 & 3.97 & 29.28 & 86.79 & 13.21 & 90.30 & 9.70 & 3.51 \\
\hline & & & Average & & $3.34 \%$ & & & & Average & & $2.50 \%$ \\
\hline
\end{tabular}

Table 6. Comparative Analysis of Accuracy Rate/Error Rate-Case Study 4

\begin{tabular}{|c|c|c|c|c|c|c|c|c|c|c|c|}
\hline \multirow{2}{*}{$\begin{array}{l}\text { Due } \\
\text { dates }\end{array}$} & \multicolumn{2}{|c|}{ Simulation (\%) } & \multicolumn{2}{|c|}{ Original PERT model (\%) } & \multirow{2}{*}{$\begin{array}{c}\text { Error rate } \\
\%\end{array}$} & \multirow{2}{*}{$\begin{array}{l}\text { Due } \\
\text { dates }\end{array}$} & \multicolumn{2}{|c|}{ Simulation (\%) } & \multicolumn{2}{|c|}{ Proposed model (\%) } & \multirow{2}{*}{$\begin{array}{c}\text { Error rate } \\
\%\end{array}$} \\
\hline & On time & Delay & On time & Delay & & & On time & Delay & On time & Delay & \\
\hline 40.33 & 29.52 & 70.48 & 32.99 & 67.01 & 3.47 & 41.17 & 30.79 & 69.21 & 32.40 & 67.60 & 1.61 \\
\hline 42.33 & 64.33 & 35.67 & 66.91 & 33.09 & 2.58 & 43.17 & 66.42 & 33.58 & 67.60 & 32.40 & 1.18 \\
\hline 43.33 & 75.35 & 24.65 & 80.95 & 19.05 & 5.60 & 44.17 & 76.80 & 23.20 & 82.00 & 18.00 & 5.20 \\
\hline \multirow[t]{2}{*}{44.33} & 88.45 & 13.55 & 90.57 & 9.43 & 4.12 & 45.17 & 87.72 & 12.28 & 91.50 & 8.50 & 3.78 \\
\hline & & & Average & & $3.94 \%$ & & & & Average & & $2.94 \%$ \\
\hline
\end{tabular}


Table 7. Comparative Analysis of Accuracy Rate/Error Rate-Case Study 5

\begin{tabular}{c|c|c|c|c|c|c|c|c|c|c|c}
\hline \multirow{2}{*}{$\begin{array}{c}\text { Due } \\
\text { dates }\end{array}$} & \multicolumn{2}{|c|}{ Simulation (\%) } & \multicolumn{2}{|c|}{ Original PERT model (\%) } & Error rate & \multirow{2}{*}{$\begin{array}{c}\text { Due } \\
\text { dates }\end{array}$} & On time & Delay & On time & Delay & \multicolumn{2}{|c|}{ Simulation (\%) } & \multicolumn{2}{c|}{ Proposed model (\%) } & \multicolumn{2}{c}{$\begin{array}{c}\text { Error rate } \\
\text { \% }\end{array}$} & & On time & Delay & On time & Delay & \\
\hline 26.17 & 28.49 & 71.51 & 31.33 & 68.67 & 2.84 & 26.68 & 28.73 & 71.27 & 30.21 & 69.79 & 1.48 \\
\hline 28.17 & 66.36 & 33.64 & 68.79 & 31.21 & 2.43 & 28.68 & 68.65 & 31.35 & 69.79 & 30.21 & 1.14 \\
\hline 29.17 & 79.59 & 20.41 & 83.60 & 16.40 & 4.01 & 29.68 & 81.14 & 18.86 & 85.01 & 14.99 & 3.87 \\
\hline 30.17 & 89.19 & 10.81 & 92.87 & 7.13 & 3.68 & 30.68 & 90.63 & 9.37 & 94.01 & 5.99 & 3.38 \\
\hline & & & Average & & $3.24 \%$ & & & & Average & & $2.47 \%$ \\
\hline
\end{tabular}

Table 8. Mean Error Rate of Cases 1-5

\begin{tabular}{c|c|c}
\hline & \multicolumn{2}{|c}{ Average error rate } \\
\hline Case study & Original PERT model (\%) & Proposed PERT model (\%) \\
\hline Case 1 & 2.48 & 1.81 \\
\hline Case 2 & 3.53 & 2.58 \\
\hline Case 3 & 3.34 & 2.50 \\
\hline Case 4 & 3.94 & 2.94 \\
\hline Case 5 & 3.24 & 2.47 \\
\hline Mean & $3.31 \%$ & $2.46 \%$ \\
\hline
\end{tabular}

CPM, activities on the longest path in the network are only identified as critical activities. However, the fact that an activity is on the critical path does not mean its criticality is high enough to influence the project completion time. This, therefore, necessitates the need to conduct sensitivity analysis which determines how critical the activity is on the critical path. Conducting sensitivity analysis helps project planners or managers to not only focus on working towards completing within the expected due date but also to pay more and critical attention to the most critical activities which are likely to delay the project so that the project milestone would be met. Fig. 6 discusses the correlation of the activities, especially the activities on the identified critical path. Correlation determines the relation of each activity and the impact on the project completion. Higher correlation coefficients indicate that the activity is the most critical and cannot be delayed. Sensitivity analysis was conducted for only the first case study. Regarding the sensitivity of the activities in case study 1 , activity $F$, which has a correlation coefficient of 0.6789 , is the most critical activity and hence, delaying this activity will delay the entire project. The next most critical activity is activity $\mathrm{D}$ with a correlation coefficient of 0.4989 . Activity $\mathrm{G}$ which is on the critical path has a correlation coefficient of only 0.2608 . This indicates that, although activity $\mathrm{G}$ is critical, its correlation coefficient is not high enough to be considered a highly critical activity to influence the project completion time, should it be delayed. Notwithstanding, attention must be paid to such near-critical activity. Activities B and C are not critical and hence, they are similar to activity $\mathrm{E}$ with no impact. Thus activities on the critical path (A-D-F) have a high correlation as compared to near-critical and the non-critical activities.

\section{Discussion of Results}

Probability network analysis for the original PERT model estimated the probability of completing the case study 1 project 1 week after the expected duration ( 23 weeks) to be $69.79 \%$, with $30.21 \%$ probability of late completion. In contrast, simulation results showed that the actual probability of completing the project

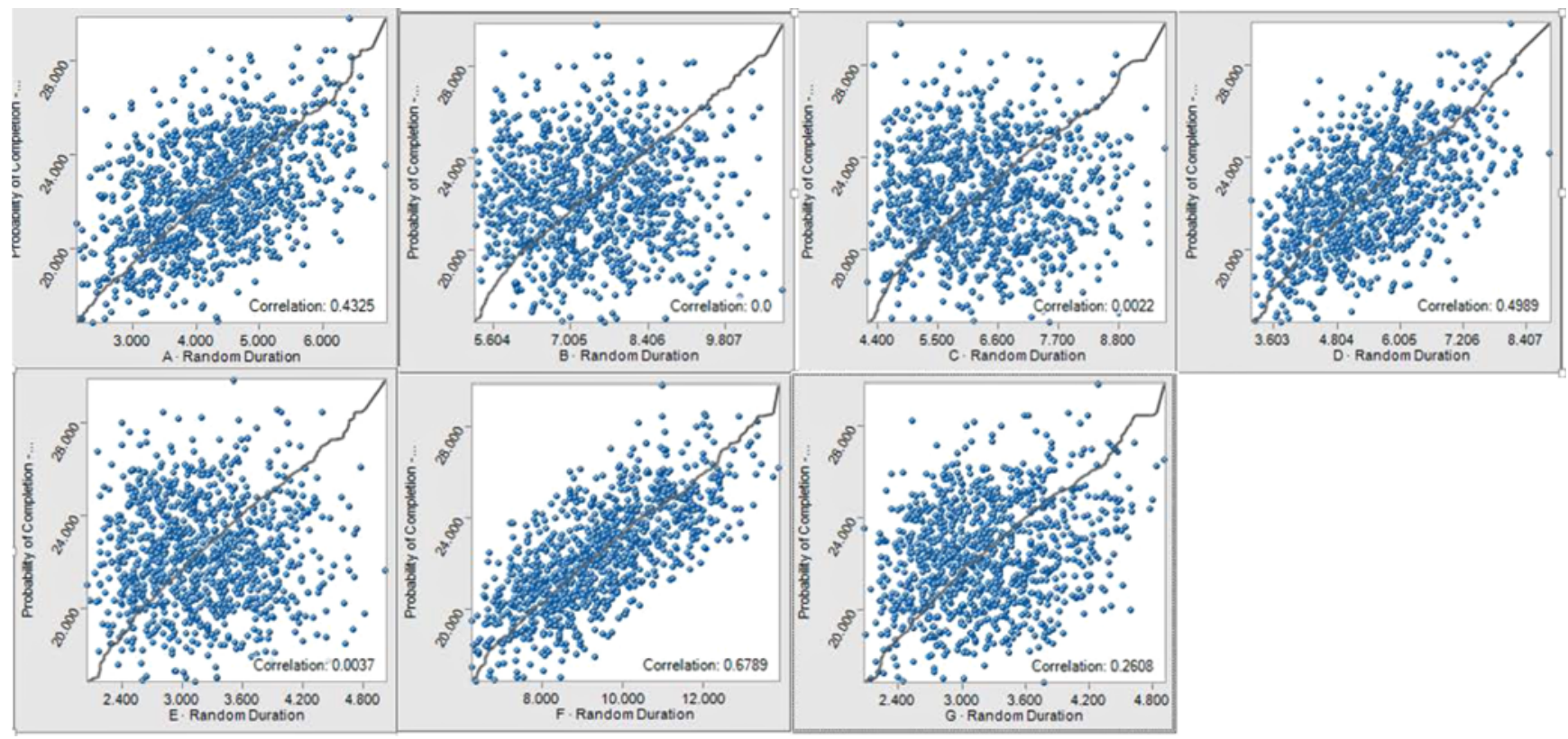

Fig. 6. Correlation of Activities in Case Study 1 
with the same assumption is $68.47 \%$, with $31.53 \%$ probability of late completion. This indicates that the original PERT model underestimates the schedule risk by approximately $1.32 \%$. At 1 week (21 weeks) the probability of completing before the expected duration is only $30.21 \%$ as compared to $33.64 \%$ in the simulation. At 2 weeks ( 24 weeks) after the expected duration, the probability of completing the project is $85.00 \%$, with $15 \%$ probability of late completion, as compared to $82.13 \%$ and $17.87 \%$, respectively in simulation; thus underestimating the schedule risk by $2.87 \%$. For 3 weeks ( 25 weeks) the probability of completion is $94 \%$, with $6 \%$ probability of late completion as compared to $91.18 \%$ probability of completion and $8.28 \%$ probability of late completion in simulation; thus underestimating the schedule risk by $2.28 \%$. However, in the case of the proposed modified PERT model, the estimated probability of completing the project 1 week after the expected duration ( 23.41 weeks) is $70.61 \%$, with $29.39 \%$ probability of late completion. Compared to the simulation results, the actual probability of completing the project with the same assumption is $70.06 \%$, with $29.94 \%$ probability of late completion. This indicates that the proposed modified PERT model underestimates the schedule risk by only $0.55 \%$. The proposed model estimated the probability of completing the project 1 week ( 21.41 weeks) before the expected duration to be $29.39 \%$, with $70.61 \%$ probability of late completion, which is close to the simulation results of $27.89 \%$ for project completion on time and $72.11 \%$ for late completion. At 2 weeks (24.41 weeks) after the expected duration, the probability of completing the project in time is $86.09 \%$ with $13.91 \%$ probability of late completion, as compared to $83.08 \%$ and $16.92 \%$ respectively in simulation; thus the schedule risk is underestimated by $3.01 \%$. This high error rate is due to the fact that the simulation stopped with a close mean of 22.235 weeks, which is 0.18 weeks less than the estimated 22.41 weeks. Nonetheless, for 3 weeks $(25.41$ weeks), the probability of completion is $94.81 \%$ and with $5.19 \%$ probability of late completion as compared to $92.65 \%$ and $7.35 \%$ respectively in simulation; thus, the schedule risk is underestimated by $2.16 \%$. Considering all the five case studies, the mean error rate for the proposed PERT model was computed as $2.46 \%$, whereas the mean error rate of the original PERT model was $3.31 \%$. Therefore, the proposed PERT model performs approximately 0.85 percentage points or $25.7 \%$ better than the original PERT model.

\section{Conclusions}

The PERT model is a useful technique that uses the three-point estimate to account for the uncertainties in the individual activities. It is used to model schedule risk. However, owing to the several assumptions that the model is based on, it can lead to underestimation of the schedule risk. The study used the original PERT model to analyze the probability of completing the project within a specified due date. The proposed modified PERT model was developed and used to model schedule risk. The probability of completing the project on time and the probability of delay were estimated for five case studies for both models. A simulation was conducted to determine the error rate for each model. The mean error rate for all the cases, cases $1-5$, was computed for both the original PERT model and the proposed modified PERT model. It was shown that the proposed modified PERT model had a better mean error rate of $2.46 \%$ as compared to $3.31 \%$ of the original PERT model. This suggests that the proposed modified PERT model performs approximately 0.85 percentage points or $25.7 \%$ better than the original PERT model. Comparing with the simulation results, the error in both models can be attributed to the fact that the PERT model only considers one path to be critical in the network. However, through verification using simulation, other paths compete to be on the critical path and the probability of any non-critical activity becoming critical effects the project completion time. Thus, the study has shown that the proposed modified PERT model can more accurately estimate the probability of completion than the original PERT model. Nevertheless, as the proposed modified model was based on certain assumptions, it is difficult to conclude with certainty that it is better than the original PERT model. However, as it yields a better result at this stage, it is hoped that using the proposed modified PERT model would aid the improvement of analysis of schedule risk in projects.

\section{References}

Avlijas, G. (2018). "Examining the value of monte carlo simulation for project time management." Management:Journal of Sustainable Business and Management Solutions in Emerging Economies, pp. 1-11, DOI: 10.7595/management.fon.2018.0004.

Ballesteros-Pérez, P. (2017). "M-PERT: Manual project-duration estimation technique for teaching scheduling basics." Journal of Construction Engineering and Management, Vol. 143, No. 9, 04017063, DOI: 10.1061/(ASCE)CO.1943-7862.0001358.

Dawson, R. J. and Dawson, C. W. (1998). "Practical proposals for managing uncertainty and risk in project planning." International Journal of Project Management, Vol. 16, No. 5, pp. 299-310, DOI: 10.1016/S0263-7863(97)00059-8.

Hajdu, M. and Bokor, O. (2014). "The effects of different activity distributions on project duration in PERT networks." Procedia Social and Behavioral Sciences, Vol. 119, No. 19, pp. 766-775, DOI: 10.1016/j.sbspro.2014.03.086.

Hajdu, M. and Bokor, O. (2016). "Sensitivity analysis in PERT networks: Does activity duration distribution matter?" Automation in Construction, Vol. 65, pp. 1-8, DOI: 10.1016/j.autcon. 2016.01.003.

Herrerías-Velasco, J. M., Herrerías-Pleguezuelo, R., and Van Dorp, J. R. (2011). "Revisiting the PERT mean and variance." European Journal of Operational Research, Vol. 210, No. 2, pp. 448-451, DOI: 10.1016/ j.ejor.2010.08.014.

Jun, H. B., Park, J. Y., and Suh, H. W. (2006). "Lead time estimation method for complex product development process." Concurrent Engineering, Vol. 14, No. 4, pp. 313-328. DOI: 10.1177/1063293X06073302.

Lee, D. E. (2005). "Probability of project completion using stochastic project scheduling simulation." Journal of Construction Engineering and Management, Vol. 131, No. 3, pp. 310-318, DOI: 10.1061/ (ASCE)0733-9364(2005)131:3(310).

Lee, D. E., Arditi, D., and Son, C. B. (2013). "The probability distribution of project completion times in simulation-based scheduling." $K S C E$ Journal of Civil Engineering, KSCE, Vol. 17, No. 4, pp. 638-645, DOI: 10.1007/s12205-013-0147-x. 
Malcolm, D. G., Roseboom, J. H., Clark, C. E., and Fazar, W. (1959). "Application of a technique for research and development program evaluation." Operations Research, Vol. 7, No. 5, pp. 646-669, DOI: 10.1287/opre.7.5.646.

Mccabe (2003). "Monte Carlo simulation for schedule risks." Proceedings of the 2003 Winter Simulation Conference, IEEE, New Orleans, LA, USA, pp. 1561-1565, DOI: 10.1109/WSC.2003.1261603.

Mishakova, A., Vakhrushkina, A., Murgul, V., and Sazonova, T. (2016). "Project control based on a mutual application of pert and earned value management methods." Procedia Engineering, Vol. 165, pp. 1812-1817, DOI: 10.1016/j.proeng.2016.11.927.

Park, H. and Cutkosky, M. R. (1999). "Framework for modeling dependencies in collaborative engineering processes." Research in Engineering Design, Vol. 11, No. 2, pp. 84-102, DOI: 10.1007/ PL00003885.

Razaque, A., Bach, C., salama, N., and Alotaibi, A. (2012). "Fostering project scheduling and controlling risk management." International Journal of Business and Social Science, Vol. 3, No. 14, pp. 118-127, DOI: arxiv.org/abs/1210.2021.

Reis, J. S. and Lucko, G. (2016). "Productivity scheduling method with maximum constraints." International Journal of Construction Management, Vol. 16, No. 1, pp. 77-93, DOI: 10.1080/15623599. 2015. 1130674.

Trietsch, D. and Baker, K. R. (2012). "PERT 21: Fitting PERT/CPM for use in the $21^{\text {st }}$ century." International Journal of Project Management, Vol. 30, No. 4, pp. 490-502, DOI: 10.1016/j.ijproman.2011.09.004.

Trietsch, D., Mazmanyan, L., Gevorgyan, L., and Baker, K. R. (2012). "Modeling activity times by the parkinson distribution with a lognormal core: Theory and validation." European Journal of Operational Research, Vol. 216, No. 2, pp. 386-396, DOI: 10.1016/j.ejor.2011.07.054.

Udoumoh, E. F. and Ebong, D. W. (2017). "A review of activity time distributions in risk analysis." American Journal of Operational Research, Vol. 7, No. 6, pp. 356-371, DOI: 10.4236/ajor.2017.76027.

Vanhoucke, M. (2012). "Dynamic scheduling: Integrating schedule risk analysis with earned value management." Measurable News, Vol. 2, pp. 11-13, DOI: 10.1007/978-3-642-16416-3_18.

van Slyke, R. M. (1963). "Monte carlo methods and the PERT problem." Operations Research, Vol. 11, No. 5, pp. 839-860, DOI: 10.1287/ opre.11.5.839. 